Vol. 12 | No. 3| Ano 2020
ARTIGO ORIGINAL

\section{SINTOMAS DO TRATO URINÁRIO INFERIOR E QUALIDADE DE VIDA NA ATENÇÃO PRIMÁRIA À SAÚDE}

\section{Symptoms of the lower urinary tract and quality of life in primary he}

Natália de Souza Duarte ${ }^{1}$, Hellem Samilles Cardoso da Costa $^{2}$, Anne Beatriz Duarte da Conceição ${ }^{3}$, Giulia Calandrini Pestana de Azevedo ${ }^{4}$, Leonardo Breno do Nascimento de Aviz 5 José Robertto Zaffalon Júnior ${ }^{6}$, Elaine da Silva Abreu ${ }^{4}$, Erica Feio Carneiro Nunes

\title{
RESUMO
}

Objetivo: Verificar a frequência de sintomas do trato urinário inferior, os fatores de risco e o impacto da Incontinencia Urinária na qualidade de vida de mulheres na Atenção Primária à Saúde. Métodos: Foram avaliadas mulheres participantes do grupo de exercícios "Mexa-se pela Vida". Os instrumentos foram uma ficha de avaliação, o "Teste das três perguntas sobre incontinência (3IQ)" e King's Health Questionnaire. Os testes estatísticos foram realizados no Bioestat 5.3 e foi utilizado um nível de significância de 5\%. Resultados: Participaram 35 voluntárias, 24 (68.5\%) apresentaram algum sintoma do trato urinário inferior. Destas, 19 (79.17\%) referiram ser afetadas com Incontinência Urinária. Não foi encontrada diferença estatisticamente significativa quanto aos fatores de risco pesquisados em mulheres com e sem sintomas do trato urinário inferior. No impacto da Incontinência Urinária na qualidade de vida, observou-se que os domínios mais afetados foram a Percepção geral de saúde e Impacto da incontinência. Conclusão: Os sintomas do trato urinário foram encontrados na maioria das mulheres, sendo o mais frequente a Incontinência Urinária, seguida de noctúria, urgência miccional, disúria e enurese. Quanto ao tipo de incontinência, a prevalência foi a de esforço. Não foram encontradas diferenças estatisticamente significantes em relação aos fatores de risco pesquisados entre as mulheres com e sem sintomas. Quanto a qualidade de vida, os domínios mais afetados são a Percepção geral de saúde e Impacto da incontinência.

Palavras-chave: Incontinência Urinária, Noctúria, Atenção Primária à Saúde, Qualidade de Vida, Mulheres.

\section{ABSTRACT}

Introduction: Symptoms of the lower urinary tract are very prevalent in women, but there are few studies within primary health care. Objective: To verify the frequency of symptoms of the lower urinary tract, the risk factors and the impact of Urinary Incontinence on the quality of life of women in Primary Health Care. Methods: Women participating in the exercise group "Move for the life". The instruments were an evaluation form, the "Test of the three questions on incontinence (3IQ)" and King's Health Questionnaire. Statistical tests were performed on Bioestat 5.3 and a significance level of 5\% was used. Results: 35 volunteers participated, $24(68.5 \%)$ had some symptom of the lower urinary tract. Of these, $19(79.17 \%)$ reported being affected with Urinary Incontinence. No statistically significant difference was found regarding the risk factors surveyed in women with and without symptoms of the lower urinary tract. In the impact of Urinary Incontinence on quality of life, it was observed that the domains most affected were the General health perception and Impact of incontinence. Conclusion: Urinary tract symptoms were found in most women, the most frequent being Urinary Incontinence, followed by nocturia, urinary urgency, dysuria and enuresis. As for the type of incontinence, the prevalence was that of effort. No statistically significant differences were found in relation to the risk factors surveyed between women with and without symptoms. As for quality of life, the domains most affected are the general health perception and impact of incontinence.

Keywords: Urinary Incontinence, Nocturia, Primary Health Care, Quality of Life, Women.

1. Fisioterapeuta. Residente em Estratégia Saúde da Família. Universidade do Estado do Pará - UEPA.

2. Fisioterapeuta. Residente em Oncologia. Universidade Federal do Pará - UFPA.

3. Graduanda do Curso de Fisioterapia. Universidade do Estado do Pará - UEPA.

4. Fisioterapeuta. Universidade do Estado do Pará - UEPA. Belém, Pará, Brasil.

5. Fisioterapeuta. Residente em Oncologia. Universidade Federal do Pará - UFPA.

6. Professor de Educação Física. Doutor em Ciências da Reabilitação.

Laboratório de Ciências Morfofuncionais. Universidade do Estado do Pará.

7. Fisioterapeuta. Doutora em Ciências da Reabilitação.

Departamento de Ciências do Movimento Humano. Universidade do Estado do Pará.

Autor de correspondência

Erica Feio Carneiro Nunes

erica@perineo.net.

DOI: doi.org/10.36692/v12n3-18 


\section{INTRODUÇÃO}

Os sintomas do trato urinário inferior (STUI) incluem sintomas de armazenamento, micção ou pós-micção ${ }^{1}$ e tem etiologia multifatorial ${ }^{2}$.

A perda involuntária de urina Incontinência Urinária (IU) - representa um dos mais frequentes STUI, podendo afetar todas as faixas etárias de ambos os sexos. Tal distúrbio é caracterizado como uma perda involuntária de urina e os fatores apontados para o grande acometimento de IU ainda não foram esclarecidos de forma adequada, no entanto, questões como aumento de peso, envelhecimento, tabagismo, hábitos alimentares, condições uroginecológicas, entre outros aspectos, podem estar relacionados ao desenvolvimento de IU, principalmente entre o público feminino ${ }^{3,4}$.

A maior predisposição de mulheres ao desenvolvimento de IU também pode estar relacionada a questões anatômicas, como diferença no comprimento uretral em relação aos homens, alteração nos mecanismos de continência decorrentes de eventos como a gestação e o parto, bem como alterações hormonais, relacionadas ao hipoestrogenismo progressivo e esgotamento dos folículos ovarianos ${ }^{5}$.

A qualidade de vida (QV) de mulheres incontinentes pode se alterar, de forma que os relacionamentos pessoal, sexual e social ficam comprometidos. Apesar da IU ser uma condição desconfortável e, muitas vezes, constrangedora, que causa limitações e/ou alterações nas atividades sociais, ocupacionais e domésticas, é observado uma certa negligência quanto a busca por tratamento de tais disfunções, visto que muitas mulheres consideram tal fator como uma condição "natural", demonstrando desconhecimento acerca do assunto ${ }^{3,6}$.

Neste cenário, a Atenção Primária à Saúde (APS) é a principal porta de entrada para o Sistema Único de Saúde (SUS) e estudos apontam que alguns profissionais atuantes apresentam conhecimento restrito/limitado sobre a IU, interferindo no diagnóstico e tratamento do problema. Além disso, essa afecção é passível de prevenção, um dos principais objetivos da $\mathrm{APS}^{7}$. No entanto, observa-se que neste contexto muitas vezes não são adotados procedimentos para prevenir ou identificar fatores de risco para IU.

Assim, tem-se a importância de estudos que busquem evidenciar a prevalência de STUI entre a população feminina atendida na APS, a fim de melhor caracterizar o perfil epidemiológico desses indivíduos e proporcionar o desenvolvimento de ações que busquem solucionar tal problemática da melhor forma possível e, consequentemente, melhorar a QV destas mulheres.

Dessa forma, este estudo tem como objetivo verificar a frequência de sintomas do trato urinário inferior, os fatores de risco e o impacto da IU na QV de mulheres na APS. 


\section{MÉTODOS}

Trata-se de um estudo transversal descritivo, aprovado pelo Comitê de Ética em Pesquisa da do Centro de Ciências Biológicas e da Saúde da Universidade do Estado do Pará (UEPA) (CAAE: 80879517.7.0000.5174, parecer 2.585.825) entre os meses de maio e agosto de 2018. Participaram do estudo mulheres do grupo de exercícios intitulado "Mexa-se pela Vida”, da Unidade Básica de Saúde Paraíso dos Pássaros, localizada em Belém do Pará. Este grupo foi criado para proporcionar exercícios físicos para a comunidade local, ocorrendo 3 vezes por semana, coordenado por uma Fisioterapeuta, um Educador Físico e uma Terapeuta Ocupacional. Os usuários praticam atividades aeróbias, de equilíbrio, resistência, alongamento, entre outras buscando a prevenção de doenças e promoção de QV.

No presente estudo foram incluídas mulheres de 18 a 80 anos que participassem ativamente do grupo, e excluídas mulheres gestantes ou com as que tivessem comprometimento cognitivo.

A coleta de dados ocorreu da seguinte forma: na primeira fase, as participantes do grupo Mexa-se pela Vida receberam esclarecimentos acerca da pesquisa e sobre o que era o assoalho pélvico e suas disfunções. Após esta fase, as participantes que aceitaram participar da pesquisa foram avaliadas conforme a ficha de avaliação criada pelos pesquisadores que continha nome, idade, estado civil e escolaridade, sintomas do trato urinário inferior, etc, e em seguida responderam os questionários “The 3 Incontinence Questions(3IQ)" e King's Health Questionnaire (KHQ) individualmente, em uma sala reservada na unidade de saúde.

O questionário 3IQ contém três questões. A primeira questão é sobre episódio de IU nos últimos três meses, a segunda questão familiariza as mulheres com os tipos de incontinência, e a terceira questão determina a categoria de incontinência: estresse, urgência, outro ou misto (estresse e urgência igualmente) ${ }^{8}$.

O KHQ, que foi respondido apenas pelas mulheres incontinentes, é composto por trinta perguntas que são arranjadas em nove domínios referentes à percepção de saúde, o impacto da incontinência, as limitações de atividades diárias, as limitações físicas, as limitações sociais, as relações pessoais, as emoções, o sono/disposição e as medidas de gravidade. Para todas as respostas são atribuídos valores numéricos, somados e avaliados por domínio. Os valores são, então, calculados por meio de fórmula matemática, obtendo-se, assim, o escore de QV, que varia de 0 a 100, considerando-se que quanto maior o número obtido, pior a $\mathrm{QV}^{9}$.

Para avaliação dos fatores de riscos em relação às disfunções urinárias foram utilizados os testes Exato de Fisher e Teste G. Para avaliação dos instrumentos de QV em relação à IU foi utilizado média e desvio padrão. Os 
testes estatísticos foram realizados no Bioestat

5.3 e foi utilizado um nível de significância de $5 \%$.

\section{RESULTADOS}

A amostra do estudo foi composta por 35 mulheres, com idade média de $53.5( \pm 8.4)$ anos, participando do grupo Mexa-se pela Vida em média a 1 ( \pm 0.43$)$ ano. A caracterização desta amostra está descrita na Tabela 1.

Tabela 1. Caracterização da amostra ( $n=35)$

\begin{tabular}{|c|c|c|}
\hline Variáveis & $\mathbf{n}$ & $\%$ \\
\hline \multicolumn{3}{|l|}{ Estado Civil } \\
\hline Solteiro & 8 & 22.9 \\
\hline Casado & 13 & 37.1 \\
\hline Divorciado & 3 & 8.6 \\
\hline Viúvo & 3 & 8.6 \\
\hline Relacionamento estável & 8 & 22.9 \\
\hline \multicolumn{3}{|l|}{ Escolaridade } \\
\hline Analfabeto & 8 & 22.9 \\
\hline Semianalfabeto & 13 & 37.1 \\
\hline Fundamental completo & 3 & 8.6 \\
\hline Ensino Médio completo & 3 & 8.6 \\
\hline Superior completo & 8 & 22.9 \\
\hline \multicolumn{3}{|l|}{ Tabagismo } \\
\hline Sim & 0 & 0.0 \\
\hline Não & 22 & 62.9 \\
\hline Anteriormente & 13 & 37.1 \\
\hline \multicolumn{3}{|l|}{ Etilismo } \\
\hline Sim & 10 & 28.57 \\
\hline Não & 16 & 45.71 \\
\hline Anteriormente & 9 & 25.71 \\
\hline \multicolumn{3}{|l|}{ História de doenças } \\
\hline Hipertensão Arterial Sistêmica (HAS) & 8 & 22.9 \\
\hline Diabetes & 3 & 8.6 \\
\hline Ambas & 3 & 8.6 \\
\hline Outros & 7 & 20.0 \\
\hline Não possui & 14 & 40.0 \\
\hline \multicolumn{3}{|l|}{ Menopausa } \\
\hline Sim & 27 & 77.14 \\
\hline Não & 8 & 22.85 \\
\hline
\end{tabular}

Na tabela 2 estão descritos os STUI urinário. Destas, 19 (79.17\%) referiram ser relatados pelas participantes onde, do total de afetadas com IU e 15 (62.50\%) Noctúria. $35,24(68.5 \%)$ apresentaram algum sintoma 
Tabela 3. Tipos de IU através do $3 \mid \mathrm{Q}(\mathrm{n}=19)$.

\begin{tabular}{|c|c|c|}
\hline Tipo de IU & $\mathrm{N}$ & $\%$ \\
\hline IUE & 8 & 42.1 \\
\hline IUM & 6 & 31.6 \\
\hline IUU & 3 & 15.8 \\
\hline Não informado & 2 & 10.5 \\
\hline Total & 19 & 100.0 \\
\hline
\end{tabular}

Legenda: IUE: Incontinência Urinária de Esforço, IUM: Incontinência Urinária Mista, IUU:

Incontinência Urinária de Urgência.

Para as pacientes que referiram IU, foi IU mais encontrado foi a IUE (42,1\%), seguida utilizado o questionário 3IQ para identificar as situações de perda de urina e classifica-las em Incontinência Urinária de Esforço (IUE), (Tabela 3). Incontinência Urinária de Urgência (IUU) e Incontinência Urinária Mista (IUM). O tipo de

Tabela 3. Tipos de IU através do $3 \mid \mathrm{Q}(\mathrm{n}=19)$.

\begin{tabular}{lcc}
\hline \multicolumn{1}{r}{ Tipo de IU } & N & $\%$ \\
\hline IUE & 8 & 42.1 \\
IUM & 6 & 31.6 \\
IUU & 3 & 15.8 \\
Não informado & 2 & 10.5 \\
\hline \multicolumn{1}{c}{ Total } & 19 & 100.0
\end{tabular}

Legenda: IUE: Incontinência Urinária de Esforço, IUM: Incontinência Urinária Mista, IUU: Incontinência Urinária de Urgência.

$\mathrm{Na}$ tabela 4 estão descritos alguns fatores de risco para STUI. Notou-se que 27 (77.14\%) das mulheres relataram estar na pósmenopausa e $13(37.14 \%)$ já estiveram em contato com tabagismo. Porém, de acordo com os testes de correlação de G e Exato de da IUM (31,6\%) e IUU (15,8\%), 2 participantes não souberam relatar a forma que perdem urina 
Tabela 4. Fatores de risco para ocorrência de sintomas do trato urinário inferior $(n=35)$.

\begin{tabular}{|c|c|c|c|c|c|}
\hline \multirow{2}{*}{ Fatores de risco } & \multicolumn{2}{|c|}{ Com sintomas (24) } & \multicolumn{2}{|c|}{ Sem sintomas (11) } & \multirow{2}{*}{ p valor } \\
\hline & $\mathbf{N}$ & $\%$ & $\mathbf{N}$ & $\%$ & \\
\hline \multicolumn{6}{|l|}{ Número de gestações } \\
\hline 0 & 2 & 8.3 & 0 & 0.0 & $0.3194^{\circ}$ \\
\hline 1 a 2 & 6 & 25.0 & 6 & 54.5 & \\
\hline 3 a 4 & 8 & 33.3 & 3 & 27.3 & \\
\hline$>=5$ & 8 & 33.3 & 2 & 18.2 & \\
\hline \multicolumn{6}{|l|}{ Número de partos vaginais } \\
\hline 0 & 8 & 33.3 & 6 & 54.5 & $0.6358^{\mathrm{a}}$ \\
\hline 1 a 2 & 7 & 29.2 & 2 & 18.2 & \\
\hline 3 a 4 & 4 & 16.7 & 2 & 18.2 & \\
\hline$>=5$ & 5 & 20.8 & 1 & 9.1 & \\
\hline \multicolumn{6}{|l|}{ Menopausa } \\
\hline Sim & 19 & 79.2 & 8 & 72.7 & $0.6851^{\mathrm{b}}$ \\
\hline Não & 5 & 20.8 & 3 & 27.3 & \\
\hline \multicolumn{6}{|l|}{ Tabagismo } \\
\hline Ex-fumante & 10 & 41.7 & 3 & 27.3 & $0.4776^{\mathrm{b}}$ \\
\hline Não & 14 & 58.3 & 8 & 72.7 & \\
\hline \multicolumn{6}{|l|}{$\begin{array}{l}\text { a Teste G } \\
\text { b Teste Exato de Fisher }\end{array}$} \\
\hline \multirow{2}{*}{\multicolumn{2}{|c|}{$\begin{array}{l}\text { Através do KHQ na análise do impacto } \\
\text { na QV das participantes, observou-se } \\
\text { domínios mais afetados são a Percepção }\end{array}$}} & \multirow{2}{*}{\multicolumn{4}{|c|}{$\begin{array}{l}\text { os menos prejudicados são Limitac } \\
\text { e Relações pessoais (Tabela 5). }\end{array}$}} \\
\hline & & & & & \\
\hline \multicolumn{6}{|c|}{ le saúde e Impacto da incontinência, e } \\
\hline \multicolumn{6}{|c|}{ Tabela 5. Qualidade de vida de pessoas acometidas com IU pelo KHQ $(n=19)$. } \\
\hline \multicolumn{2}{|l|}{ Domínios } & Média & \multicolumn{3}{|c|}{ Desvio padrão } \\
\hline \multicolumn{2}{|l|}{ Percepção geral de saúde } & 43.42 & \multicolumn{3}{|c|}{ \pm 23.33} \\
\hline \multicolumn{2}{|l|}{ Impacto da incontinência } & 41.78 & \multicolumn{3}{|c|}{ \pm 32.9} \\
\hline \multicolumn{2}{|c|}{ Limitações de atividades diárias } & 17.36 & \multicolumn{3}{|c|}{ \pm 20.37} \\
\hline \multicolumn{2}{|l|}{ Limitações físicas } & 14.73 & \multicolumn{3}{|c|}{ \pm 23.3} \\
\hline \multicolumn{2}{|l|}{ Limitações sociais } & 9.57 & \multicolumn{3}{|c|}{ \pm 15.61} \\
\hline \multicolumn{2}{|l|}{ Relações pessoais } & 7.84 & \multicolumn{3}{|c|}{ \pm 20.94} \\
\hline \multicolumn{2}{|l|}{ Emoções } & 20.26 & \multicolumn{3}{|c|}{ \pm 22.68} \\
\hline \multicolumn{2}{|l|}{ Sono/disposição } & 31.36 & & \pm 38.43 & \\
\hline Medidas de gravidade & & 28.66 & & \pm 22.07 & \\
\hline
\end{tabular}




\section{DISCUSSÃO}

O objetivo desta pesquisa foi verificar a prevalência de IU, fatores de risco para disfunções urinárias e o impacto da IU na QV de mulheres na APS.

Neste estudo observou-se que a maioria das participantes apresentaram pelo menos um sintoma urinário com destaque para IU, em especial a IUE. A IUE também foi a mais frequente em outros estudos com mulheres atendidas na APS $^{10,11}$.

Nesta pesquisa as participantes tinham média de idade de 53,5 anos e encontravamse na pós-menopausa, que são fatores de risco para IUE10, ${ }^{11}$. Um estudo11 relata que a faixa etária com maior queixa de IU encontra-se entre 40-59 anos, sendo que nesta fase 30 a $60 \%$ das mulheres podem sofrer $\mathrm{IU}^{12}$.

A menopausa predispõe à STUI pelas alterações hormonais, desencadeando uma queda do nível de estrogênioe consequentemente comprometendo a função do trato urinário inferior ${ }^{13}$. O tabagismo também se mostra como um dos fatores de risco culminando com a diminuição de estrogênio associado a tosse crônica ${ }^{14}$.

O perfil amostral de estudo expôs que a maioria das mulheres eram casadas, com ensino fundamental incompleto, e relataram consumir ou já ter consumido álcool. Tal perfil já foi encontrado em outros estudos que verificaram que as participantes mais acometidas pela IU eram brancas, casadas, possuindo grau de escolaridade até o nível fundamental, com renda familiar de 1 a 2 salários-mínimos, não tabagistas e não etilistas ${ }^{15,16}$.

Outro autor ${ }^{17}$ relata que o álcool pode desempenhar um fator relevante na gênese dos STUI, tanto por seu efeito diurético, quanto por uma possível irritação direta da mucosa vesical.

No que concerne as comorbidades, algumas mulheres relataram possuir Hipertensão Arterial Sistêmica, e se sabe que para que haja o controle dessa patologia vários medicamentos são utilizados, como os diuréticos, que causam um aumento da frequência urinária. Além deste, os betabloqueadores e os bloqueadores dos canais de cálcio são considerados os fármacos anti-hipertensivos mais associados com os distúrbios urinários ${ }^{18}$.

Demais fatores de risco para IU encontrados neste estudo não foram estatiscamente significantes, sendo eles: número de gestações, número de partos vaginais, apesar de que no grupo de mulheres com queixas urinárias o número de filhos ter sido maior, indicando uma possível tendência. Uma pesquisa com mulheres atendidas em uma Unidade Básica de Saúde mostrou a associação entre o maior número de gestações e o desenvolvimento de IU, porém sem associação com os partos vaginais ${ }^{10}$. Isso pode ocorrer devido a ação da crescente pressão do útero e do peso fetal nos músculos do assoalho pélvico associado às alterações hormonais que levam 
à redução da força e da função de apoio do esfíncter uretral ${ }^{14}$.

O parto vaginal é geralmente apontado como um fator risco para o desenvolvimento de IUE, devido à possíveis traumas no assoalho pélvico decorrentes do parto. No entanto, isso depende da forma que o mesmo for conduzido $^{19}$. Além disso, todas as mulheres são praticantes de atividades físicas, a em média 1 ano, e a literatura também mostra a associação entre a pratica de atividade física e a $\mathrm{IU}^{20}$.

A noctúria foi a segunda queixa mais encontrada neste estudo, que pode ser caracterizada como o ato de acordar à noite uma vez ou mais para urinar. Sua prevalência foi de $56.9 \%$ em 1472 mulheres chinesas pesquisadas, sendo o principal sintoma do trato urinário inferior, porém foi apontado como o sintoma menos incomodo, diferente da IU e enurese noturna, que foram os apontados como os mais desagradáveis ${ }^{21}$. Um estudo oriental, já considerando a noctúria como pelo menos dois episódios por noite, a encontrou também como sintoma principal do trato urinário inferior, atingindo $37 \%$ das 4209 mulheres pesquisadas $^{22}$. Outro estudo com 66 mulheres brasileiras atendidas na APS encontrou a noctúria em $80 \%$ das que apresentavam $\mathrm{IU}^{23}$.

Diante das diversas alterações sociais, pessoais e emocionais das mulheres, frente a perda urinária e fecal, medidas de avaliação da QV têm sido recomendadas ${ }^{24}$. A International Continency Society sugere a utilização de questionários para avaliação da QV, pois, os métodos que são utilizados geralmente na prática clínica tratam-se de parâmetros estritamente biomédicos e que não contemplam o impacto que tal condição acarreta no cotidiano dessas mulheres ${ }^{25}$.

Quanto à QV, estudos verificaram que a IU determina um impacto negativo na vida das mulheres, demonstrando prejuízos importantes nos scores dos domínios do KHQ e que mulheres com IUE geralmente tem pior QV quando comparada a IUU e IUM ${ }^{16,26,27,28}$. Apesar destes dados, parte dos profissionais da saúde negligencia a IU, descuidando de investigar e consequentemente de fornecer orientações e encaminhamentos necessários à condução de tratamentos e cuidados adequados ${ }^{10}$.

As dificuldades encontradas neste estudo se referem ao período da coleta de dados que coincidiu em parte com as férias do grupo, favorecendo a diminuição do número amostral, além disso, o fator de baixa escolaridade limitou o entendimento das participantes em relação aos questionários. Uma outra limitação da pesquisa foi haver mais de um pesquisador realizando a coleta de dados, o que pode ter gerado controvérsia quanto a interpretação das perguntas.

Sugere-se a realização de mais estudos verificando a prevalência de outras disfunções do assoalho pélvico nesse público e pesquisas que busquem estabelecer um plano preventivo 
ou curativo para essas disfunções que muitas vezes são negligenciadas na APS.

\section{CONCLUSÃO}

Os STUI foram encontrados na maioria das mulheres estudadas, sendo o mais frequente a IU, seguida de noctúria, urgência miccional, disúria e enurese. Quanto ao tipo de IU, a pravalência foi de IUE. Não foram encontradas diferenças estatisticamente significantes em relação aos fatores de risco pesquisados entre as mulheres com e sem sintomas do trato urinário inferior. Quanto a QV, observou-se que os domínios mais afetados são a Percepção geral de saúde e Impacto da incontinência. Esperase que este estudo sirva como embasamento para futuros direcionamentos ao tratamento dessas afecções no SUS e que mais pesquisas pesquisas sejam feitas a fim de estudar melhor o comportamento dos sintomas urinários no publico feminino atendido na APS.

\section{REFERÊNCIAS}

1. Coyne KS, Sexton CC, Thompson CL, Milsom I, Irwin D, Kopp ZS et al. The prevalence of lower urinary tract symptoms (LUTS) in the USA, the UK and Sweden: results from the Epidemiology of LUTS (EpiLUTS) study. BJU Int. 2009 Aug;104(3):352-60. doi: 10.1111/j.1464-410X.2009.08427.x.

2. Gravas S, Kyriazis I, Klausner AP. Lower Urinary Tract Symptoms Including Bladder Outlet Obstruction: What's New in Diagnostics? Eur Urol Focus. 2018 Jan;4(1):14-16. doi: 10.1016/j.euf.2018.04.004.

3. Da silva JCP, Soler ZASG, Domingueswysocki A. Fatores associados à incontinência urinária em mulheres submetidas ao exame urodinâmico. Rev Esc Enferm USP. 2017, 51:03209.

4. Martinez MA, Kraievski ES. O advento da Fisioterapia no tratamento da incontinência urinária de esforço. Rev Conexão Eletrônica, Três Lagoas. 2017,14(1): 190-9.

5. Almeida CJ, Ramos GCF, Barbosa ATF, Medeiros
SM, Lima CA, Costa FM et al. Prevalência e fatores associados à incontinência urinária em idosos não institucionalizados. Cad Saude Colet. 2017, 25(3).

6. Knorst MR, Royer CS, Basso DMS, Russo JS, Guedes RG, Resende TL. Avaliação da qualidade de vida antes e depois de tratamento fisioterapêutico para incontinência urinária. Braz J Phys Ther. 2013, 20(3): 204-209.

7. Tomasi AV, Santos SMA, Honório GJS, Locks MOH. Incontinência urinária em idosas: práticas assistenciais e proposta de cuidado âmbito da atenção primária de saúde. Texto Contexto Enferm, 2017; 26(2).

8. Brown JS, Bradley CS, Subak LL, Richter HE, Kraus SR, Brubaker L, et al. The sensitivity and specificity of a simple test to distinguish between urge and stress urinary incontinence. Ann Intern Med. 2006;144(10):715-723.

9. Fonseca ESM, Camargo ALM, Castro RA, Sartori MGF, Fonseca MCM, Lima GR. Validação do questionário de qualidade de vida (King's Health Questionnaire) em mulheres brasileiras com incontinência urinária. Rev Bras Ginecol Obstet. 2005;27(5):235-42.

10. Benício CDAV, Luz MHBA, Lopes MHBM, Carvalho NAR. Incontinência urinária: prevalência e fatores de risco em mulheres em uma Unidade Básica de Saúde. Estima. 2016; 14(4):161-168.

11. Oliveira TM, Valdez FML, Lima KES, Magalhães MS, Abdon APV, Bezerra IN. Prevalência de Incontinência Urinária e fatores associados em mulheres no climatério em uma unidade de atenção primária à saúde. Rev Bras Promoç Saúde. 2015; 28(4): 606-612.

12. Berbam LW. Exercícios de Kegel e ginástica hipopressiva como estratégia de atendimento domiciliar no tratamento da incontinência urinária feminina: relato de caso [trabalho de conclusão de curso]. Ijuí: Universidade Regional do Noroeste do Estado do Rio Grande do Sul, Curso de Fisioterapia; 2011.

13. Robinson D, Cardozo L. Estrogens and the lower urinary tract. Neurourol Urodyn. 2011; 30:754-7.

14. Sangsawang B. Risk factors for the development of stress urinary incontinence during pregnancy in primigravidae: a review of the literature. Eur J Obstet Ginecol Reprod Biol. 2014;178: 27-34

15. Silva AI, Almeida C, Aguiar H, Neves M, Teles MJ. Prevalência e impacto da incontinência urinária na qualidade de vida da mulher. Rev Port Med Geral Fam. 2013; 29:364-76.

16. Berquó MS, Ribeiro MO, Amaral RG. Qualidade de vida de mulheres portadoras de incontinência urinária antes e após a fisioterapia realizada no hospital materno infantil de Goiânia goiás. Rev Cien Escol Estad Saud Publ Cândido Santiago RESAP. 2016;2(2):104-122

17. Zerati M, Morais HCF, Ferreira CHJ. Alterações do Estilo de Vida: O Primeiro Passo? In: Palma P, editor. Urofisioterapia: aplicações clínicas das técnicas fisioterapêuticas nas disfunções miccionais e do assoalho pélvico. Campinas: Personal Link Comunicações. 2009;175-86.

18. Berlezi EM, Fiorin AAM, Bilibio PVF, Kirchner RM, Oliveira KR. Estudo da incontinência Urinária em mulheres climatéricas usuárias de medicação anti- 
hipertensiva. Rev. Bras. Geriatr. Gerontol. Rio de Janeiro. 2011; 14(10):415-423.

19. Silva JCP, Soler ZASG, Wysocki AD. Fatores associados à incontinência urinária em mulheres submetidas ao exame urodinâmico. Rev Esc Enferm USP. 2017; 51: e03209. https://doi.org/10.1590/S1980$220 \times 2015048003209$.

20. Mourão LF, Luz MHBA, Marques ADB, Benício CDAV, Nunes BMVT, Pereira AFM. Caracterização e fatores de risco de incontinência urinária em mulheres atendidas em uma clínica ginecológica. Estima. 2017; 15(2):82-91.

21. Wang $\mathrm{Y}, \mathrm{Hu} \mathrm{H}, \mathrm{Xu} \mathrm{K}$, Wang X, Na Y, Kang X. Prevalence, risk factors and the bother of lower urinary tract symptoms in China: a population-based survey. Int Urogynecol J. 2015;26(6):911-919. doi: 10.1007/ s00192-015-2626-8.

22. Chapple C, Castro-Diaz D, Chuang Y-C, Lee KS, Liao L, Liu SP et al. Prevalence of Lower Urinary Tract Symptoms in China, Taiwan, and South Korea: Results from a Cross-Sectional, Population-Based Study. Advances in Therapy. 2017;34(8):1953-1965. doi:10.1007/s12325-017-0577-9.

23. Faria CA, Menezes AMN, Rodrigues AO, Ferreira ALV, Bolsas CN. Incontinência urinária e noctúria: prevalência e impacto sobre qualidade de vida em idosas numa Unidade Básica de Saúde. Rev bras geriatr gerontol [Internet]. 2014 Mar [cited 2018 May 31]; 17(1):17-25. Available from: http://www. scielo.br/scielo.php?script $=$ sci_arttext\&pid $=\mathrm{S} 1809$ 98232014000100017\&lng=en. http://dx.doi. org/10.1590/S1809-98232014000100003.

24. Vasconcelos CTM, Vasconcelos Neto JA, Bezerra LRPS, Augusto KL, Karbage SAL, Frota IPR, et al. Disfunções do assoalho pélvico: perfil sóciodemográfico e clínico das usuárias de um ambulatório de uroginecologia. Revista Eletrônica Gestão \& Saúde. 2013; 4(1) :1202-1216

25. Haylen BT, Ridder D, Freeman RM, Swift EE, Berghmans B, Lee J, Monga A, Petri E, Rizk DE, Sand PK, Schaer GN. An International Urogynecological Association (IUGA) / International Continence Society (ICS) joint report on the terminology for female pelvic floor dysfunction. Neurourology and Urodynamics. 2010; 29(1):4-20.

26. Faria CA, Moraes JR, Monnerat BR, Verediano KA, Hawerroth PA, Fonseca SC. Impacto do tipo de incontinência urinária sobre a qualidade de vida de usuárias do Sistema Único de Saúde no Sudeste do Brasil. Rev Bras Ginecol Obstet. 2015; 37(8):374-80

27. Bomfim IQ, Soutinho RSR, Araújo EN.Comparação da Qualidade de Vida das Mulheres com Incontinência Urinária Atendidas no Sistema de Saúde Pública e Privada. Unopar Cient Ciênc Biol. Saúde. 2014; 16(1):1924.

28. Fernandes S, Coutinho EC, Duarte JC, Nelas PAB, Chaves CMCB, Amaral O. Qualidade de vida em mulheres com Incontinência Urinária. Rev Enf Ref. [Internet]. 2015 Jun [citado 2019 Set 09];(5):93-99. Disponível em: http://www.scielo.mec.pt/scielo.php?script=sci_ arttext\&pid $=$ S0874-02832015000200011\&lng $=$ pt. http://dx.doi.org/10.12707/RIV14042.

OBSERVAÇÃO: Os autores declaram não existir conflitos de interesse de qualquer natureza. 